Check for updates

Cite this: J. Mater. Chem. A, 2017, 5 , 21836

Received 10th July 2017

Accepted 14th September 2017

DOI: $10.1039 / c 7 t a 05967 a$

rsc.li/materials-a

\section{Elucidating the role of the hole-extracting electrode on the stability and efficiency of inverted $\mathrm{CsSnI}_{3} / \mathrm{C}_{60}$ perovskite photovoltaics $\uparrow$}

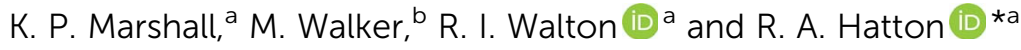

The correlation between the stability of thin films of black (B) $-\gamma \mathrm{CsSnl}_{3}$ perovskite in ambient air and the choice of supporting substrate is examined for the substrates: (i) soda-lime glass; (ii) indium tin oxide (ITO) glass; (iii) copper iodide (solution processed)/ITO glass; (iv) poly(3,4-ethylenedioxythiophene):poly(styrenesulfonate) (PEDOT:PSS)/ITO glass; (v) and an optically thin $(8 \mathrm{~nm})$ gold film electrode. The performance of (ii) $-(\mathrm{v})$ as the hole-extracting electrode in inverted photovoltaic (PV) devices with a simple bilayer architecture is compared for a test condition of 1 sun continuous solar illumination in air. $\mathrm{CsSnl}_{3}$ film stability is shown to depend strongly on the density of pinholes and grain boundaries, although not on the preferred $\mathrm{CsSnl}_{3}$ crystallite orientation. Solution processed $\mathrm{Cul}$ is shown to be unsuitable as a hole-transport layer (HTL) for inverted $\mathrm{CsSn}_{3} \mathrm{PV}$ devices because it is almost completely displaced by the $\mathrm{CsSnl}_{3}$ precursor solution during the spin coating process, and its large ionisation potential is poorly matched to the valence band edge of $\mathrm{CsSnl}_{3}$. Devices using an ITO (or Au) hole-extracting electrode with no HTL are found to be more stable than those using the archetypal HTL; PEDOT:PSS. Spectroscopic analysis of the $\mathrm{CsSnl}_{3}$ layer recovered from PV devices after 24 hours testing in ambient air (with no device encapsulation) shows that $\leq 11 \%$ of the $\mathrm{CsSnl}_{3}$ film thickness is oxidised to $\mathrm{Cs}_{2} \mathrm{Snl}_{6}$ due to air ingress, which shows that the deterioration in device efficiency under continuous illumination does not primarily result from a reduction in the light absorption capability of the perovskite film due to $\mathrm{CsSnl}_{3}$ oxidation. Additionally it is shown that $\mathrm{SnCl}_{2}$ added during $\mathrm{CsSnl}_{3}$ film preparation reduces the extent of $\mathrm{p}$-type self-doping of the perovskite film and serves as an $n$-type dopant for the adjacent evaporated $\mathrm{C}_{60}$ electron transport layer.

\section{Introduction}

Lead halide perovskites have been intensively researched for use in photovoltaics (PVs) since $2009,{ }^{1}$ and have already achieved a certified power conversion efficiency $(\eta)$ of $22.1 \%$, which is approaching that of conventional silicon PVs. ${ }^{2}$ However, the possibility of $\mathrm{Pb}$ contamination of the environment as a result of failure of the device encapsulants and/or improper disposal at the end of life is a serious concern for commercial exploitation because $\mathrm{Pb}$ is known to have high toxicity and can accumulate in the food chain..$^{3-6}$ This problem is particularly acute for $\mathrm{Pb}$ perovskites because they decompose in the presence of moisture to form water soluble toxic lead compounds such as $\mathrm{PbI}_{2}$ and $\mathrm{PbCO}_{3}{ }^{7,8}$ Consequently there is a need to identify perovskite semiconductors suitable for PVs that use lower toxicity replacements for $\mathrm{Pb}$. Tin halide perovskites are one such possibility, and are also particularly attractive because of

${ }^{a}$ Department of Chemistry, University of Warwick, Coventry, CV4 7AL, UK. E-mail: Ross.Hatton@warwick.ac.uk

${ }^{b}$ Department of Physics, University of Warwick, Coventry, CV4 7AL, UK

$\dagger$ Electronic supplementary information (ESI) available. See DOI: 10.1039/c7ta05967a their lower band gaps than lead analogues (typically 1.3-1.4 eV), which are ideal for single junction PVs, very low exciton binding energies $(<20 \mathrm{meV})$ and very large charge carrier mobilities (1-100 $\mathrm{cm}^{2} \mathrm{~V}^{-1} \mathrm{~s}^{-1}$ ) which collectively bode very well for the possibility of achieving a $\eta$ comparable to that of $\mathrm{Pb}$ perovskites. ${ }^{9-12}$ However, Sn perovskites have not been widely researched because of the instability of $\mathrm{Sn}$ in the $2+$ oxidation state, which is easily converted to the $4+$ state in the presence of moisture and oxygen. ${ }^{9,13}$ Additionally, Sn halide perovskites are susceptible to the formation of a high density of Sn vacancy defects which can severely limit the device fill factor (FF) and open-circuit voltage $\left(V_{\mathrm{oc}}\right) \cdot{ }^{\mathbf{1 2 , 1 4}}$

One approach to minimising tin vacancy density in Sn perovskites is to synthesise the perovskite in a Sn-rich environment, ${ }^{12,14-16}$ although for device applications consideration must also be given to the fate of the compound supplying the excess $\mathrm{Sn}$ once the perovskite has formed, since the two components inevitably form a complex two-phase film..$^{12,17,18} \mathrm{We}$ have recently shown that $\mathrm{SnCl}_{2}$ is particularly effective as a source of excess $\mathrm{Sn}$ when synthesising $\mathrm{CsSnI}_{3}$ perovskite, and is pushed to the surface of the $\mathrm{CsSnI}_{3}$ crystallites where it serves the additional role of doping the adjacent phenyl- $\mathrm{C}_{61}$-butyric acid methyl ester (PCBM) when the perovskite film is integrated 
into an inverted device architecture. ${ }^{17}$ Importantly $\mathrm{SnCl}_{2}$ does not significantly increase the toxicity of the material system; indeed it is used an antioxidant in food, known as E512 in Europe. ${ }^{19}$

The stability of lead perovskite PVs is known to depend strongly dependent on the choice of materials used for the extraction of holes from the perovskite layer, particularly in inverted device architectures, where it not only ensures optimal interfacial energy level alignment for selective extraction of holes but is also a key determinant of perovskite film structure and morphology. ${ }^{20-22}$ Herein we report the results of a study focused on how the stability of inverted $\mathrm{CsSnI}_{3} \mathrm{PV}$ devices with the structure: hole-extracting window electrode $\mid \mathrm{CsSnI}_{3}: \mathrm{SnCl}_{2}$ $\left|\mathrm{C}_{60}\right|$ bathocuproine (BCP)|Al depends on the choice of holeextracting substrate. The hole-extracting electrode materials compared are: (i) ITO coated glass, because it is the most widely used transparent electrode and is sufficiently smooth to be used in simple bilayer perovskite PV device architecture without an additional layer to smooth the electrode surface. This system serves as the benchmark against which the others are compared; (ii) ITO/copper iodide (CuI), because CuI has recently been used for both $\mathrm{Pb}^{23-25}$ and $\mathrm{Sn}^{15}$ based PPVs, and is reported to be a very stable hole-transport layer (HTL) for $\mathrm{Pb}$ perovskite PV applications; ${ }^{23-25}$ (iii) ITO/poly(3,4-ethylenedioxythiophene):poly(styrenesulfonate) (PEDOT:PSS), because it is the most widely used HTL used in inverted $\mathrm{Pb}$ perovskite PV research $^{26-28}$ and, due to the very high doping level PEDOT:PSS can be regarded as a synthetic metal; (iv) an optically thin $(8 \mathrm{~nm}$ thick) $\mathrm{Au}$ electrode supported on glass fabricated using the molecular adhesive layer method, ${ }^{29}$ because $\mathrm{Au}$ is widely used in perovskite PV research due to its high stability and, in the context of this study, serves as a chemically stable and structurally well-defined ${ }^{29,30}$ model electrode. To date the stability of Sn PVs towards complex conditions (i.e. constant 1 sun illumination, moisture, elevated temperature) have been sparsely reported, ${ }^{18,31-33}$ and to our knowledge this is the first report comparing the stability of inverted Sn perovskite PV with the choice of hole-extracting electrode/HTL.

\section{Experimental}

\section{B- $\gamma \mathrm{CsSnI}_{3}$ film deposition}

In a dry nitrogen filled glove box $\left(<1 \mathrm{ppm}_{2} \mathrm{O}\right.$ and $\left.\mathrm{O}_{2}\right), \mathrm{SnI}_{2}$, CsI and $\mathrm{SnCl}_{2}$ were added to a vial in a $1: 1: 0.1$ ratio. Anhydrous $N, N$-dimethylformamide (DMF) was added to make the solution up to $8 \mathrm{wt} \%$ by total mass of solids, and left overnight to allow the material to dissolve. One drop of solution was cast onto a substrate spinning at $4000 \mathrm{rpm}$, and allowed to spin for 60 seconds to form $\mathrm{CsSnI}_{3}: \mathrm{SnCl}_{2}$ films with an intense dark red/brown colour. The films formed while spinning as the solvent evaporates.

\section{Photovoltaic device fabrication}

ITO coated glass slides were cleaned by immersing in acetone and placing in an ultrasonic bath for 10 minutes. This process was repeated using high purity water containing a few drops of
Decon 90 surfactant, then with water only and finally isopropanol. The slides were then suspended in boiling acetone vapour for 10 seconds and dried in a flow of nitrogen, before $\mathrm{UV} / \mathrm{O}_{3}$ treatment for 15 minutes using a 185/254 nm light source, before being transferred into the glovebox for device fabrication.

CuI was spin cast from $50 \mathrm{mg} \mathrm{ml}^{-1}$ di-n-propylsulfide solutions at $2000 \mathrm{rpm}$, and left in the glovebox for 30 minutes to dry. $\mathrm{CsSnI}_{3}$ was spin cast at $4000 \mathrm{rpm}$ from $8 \mathrm{wt} \%$ DMF solution.

$\mathrm{C}_{60}$ was deposited by thermal evaporation at $\sim 500{ }^{\circ} \mathrm{C}$ at a rate of 0.5 to $1 \AA \mathrm{s}^{-1}$ to a thickness of $40 \mathrm{~nm}$. Bathocuproine (BCP) was thermally evaporated at $\sim 140{ }^{\circ} \mathrm{C}$ at a rate of $\sim 0.5 \AA^{\circ} \mathrm{s}^{-1}$ to a thickness of $6 \mathrm{~nm}$. Al was evaporated at a rate of $1 \AA \mathrm{s}^{-1}$ to a thickness of $50 \mathrm{~nm}$.

\section{$8 \mathrm{~nm}$ Au electrode fabrication}

$\mathrm{Au}$ films with a thickness of $8 \mathrm{~nm}$ were fabricated on glass substrates using the procedure previously reported by our group. ${ }^{29}$ In brief, glass microscope slides were cleaned and exposed to vapour of 3-aminopropyltrimethoxysilane and 3-mercaptopropyltrimethoxysilane in a desiccator at 40 mbar. The Au films were $\mathrm{UV} / \mathrm{O}_{3}$ treated for 15 minutes immediately prior to $\mathrm{CsSnI}_{3}$ deposition.

\section{X-ray diffraction (XRD)}

$\mathrm{XRD}$ was performed on thin films of $\mathrm{CsSnI}_{3}$ prepared from $8 \mathrm{wt} \%$ (total solids) DMF solution deposited onto a substrate spinning at $4000 \mathrm{rpm}$ for $60 \mathrm{~s}$. Scans were recorded under a flow of nitrogen using a $\mathrm{Cu} \mathrm{K} \alpha_{1 / 2}$ source in $\theta-\theta$ mode on a Bruker D8 Advance powder diffractometer equipped with an Anton-Paar HTK900 gas chamber. The measured XRD patterns were corrected for height offset (due to the height of the glass substrate) by calibrating the $2 \theta$ scale with reference to the expected peak positions for pure B- $\gamma \mathrm{CsSnI}_{3}$. Simulated diffraction patterns were calculated using the program, Mercury 3.5.1 (ref. 35) using crystallographic information files from the Inorganic Crystal Structure Database (ICSD).

\section{Photoelectron spectroscopy}

X-ray photoelectron spectroscopy (XPS) and ultra-violet photoelectron spectroscopy (UPS) were performed on samples supported on Au coated glass using a Kratos AXIS Ultra DLD at a base pressure of $5 \times 10^{-11}$ mbar. Samples were loaded into the spectrometer using an air tight sample transfer arm that enables transfer from the glovebox to the spectrometer without exposure to air. Au electrodes were $\mathrm{UV} / \mathrm{O}_{3}$ treated for 15 minutes before sample deposition to achieve compact perovskite films. For XPS analysis the sample was excited with X-rays from a monochromated $\mathrm{Al} \mathrm{K} \alpha$ source $(h \nu=1486.7 \mathrm{eV})$, with the photoelectrons being detected at a $90^{\circ}$ take-off angle. Peak fitting was performed using the CasaXPS package, ${ }^{36}$ incorporating Voigt (mixed Gaussian-Lorentzian) line shapes and a Shirley background. UPS was performed in the same vacuum system as for XPS using a $21.22 \mathrm{eV}$ He $1 \alpha$ light source. 


\section{Electronic absorption spectroscopy}

Ultraviolet/visible/near-infrared spectra were measured for optically thin films of $\mathrm{CsSnI}_{3}$ on glass or ITO glass substrates.

\section{Optical field and electronic absorption simulations}

The Essential Macleod V 9.7 (ref. 34) software simulation package was used for simulating light intensity in devices.

\section{Contact potential measurement}

Work function measurements were performed using a Kelvin probe referenced to freshly cleaved highly oriented pyrolytic graphite in a nitrogen-filled glovebox co-located with the spin coater and thermal evaporator.

\section{Atomic force microscopy (AFM)}

AFM was performed on a Veeco Multimode using films on glass substrates. Analysis was performed using the program WSxM $4.0 .^{37}$

\section{Scanning electron microscopy (SEM)}

SEM imaging was performed using a Zeiss SUPRA 55VP field emission gun SEM and crystallite size analysis was performed using the Image ${ }^{38}$ program.

\section{Results \& discussion}

\section{B- $\gamma$ CsSnI $_{3}$ film structure and stability on different substrates}

The perovskite B- $\gamma \mathrm{CsSnI}_{3}$ is known to degrade in ambient air in a two-stage process; firstly into the one dimensional yellow phase of $\mathrm{CsSnI}_{3}\left(\mathrm{Y}^{-} \mathrm{CsSnI}_{3}\right)$ with the same chemical composition upon reaction with moisture and then into the zerodimensional $\mathrm{Sn}$ (Iv) salt $\mathrm{Cs}_{2} \mathrm{SnI}_{6}$ upon reaction with oxygen ${ }^{\mathbf{9}, \mathbf{1 3}, 17}$ the latter of which has an absorption coefficient across the visible spectrum $\sim 10 \times$ smaller than that of $\mathrm{B}-\gamma \mathrm{CsSnI}_{3}{ }^{11}$ Exploiting the large reduction in absorption strength upon oxidation to $\mathrm{Cs}_{2} \mathrm{SnI}_{6}$, the evolution of the absorption spectrum of thin films of $\mathrm{CsSnI}_{3}$ supported on the different substrates was used to follow the oxidation of $\mathrm{CsSnI}_{3}$ films in air: Fig. 1(a)-(c).

It is evident from Fig. 1 that the stability of $\mathrm{CsSnI}_{3}: \mathrm{SnCl}_{2}$ films decreases in the order ITO glass $>$ glass $\approx$ CuI/ITO glass $>$ PEDOT:PSS/ITO glass > Au. Whilst glass is not useful as an electrode it is a simple transparent reference substrate against which the optical properties of the perovskite film on the other more complex substrates can be compared. The spectrum of $\mathrm{CsSnI}_{3}: \mathrm{SnCl}_{2}$ on ITO includes a broad local minimum at $\sim 400 \mathrm{~nm}$ that is not a feature of $\mathrm{CsSnI}_{3}$ films on all of the other substrates; Fig. 1(a) and (b) and ESI Fig. S1(a)-(c), $\dagger$ although optical modelling reveals that this feature results from an optical interference effect (Fig. S2 $\dagger$ ) rather than from a difference in electronic structure.

The stability of $\mathrm{CsSnI}_{3}: \mathrm{SnCl}_{2}$ films supported on different HTL/electrodes towards air-oxidation is inevitably a complex function of: (i) the intrinsic stability of the perovskite film (which strongly dependents on its morphology, including the crystallite size and orientation); (ii) the density of pinholes and
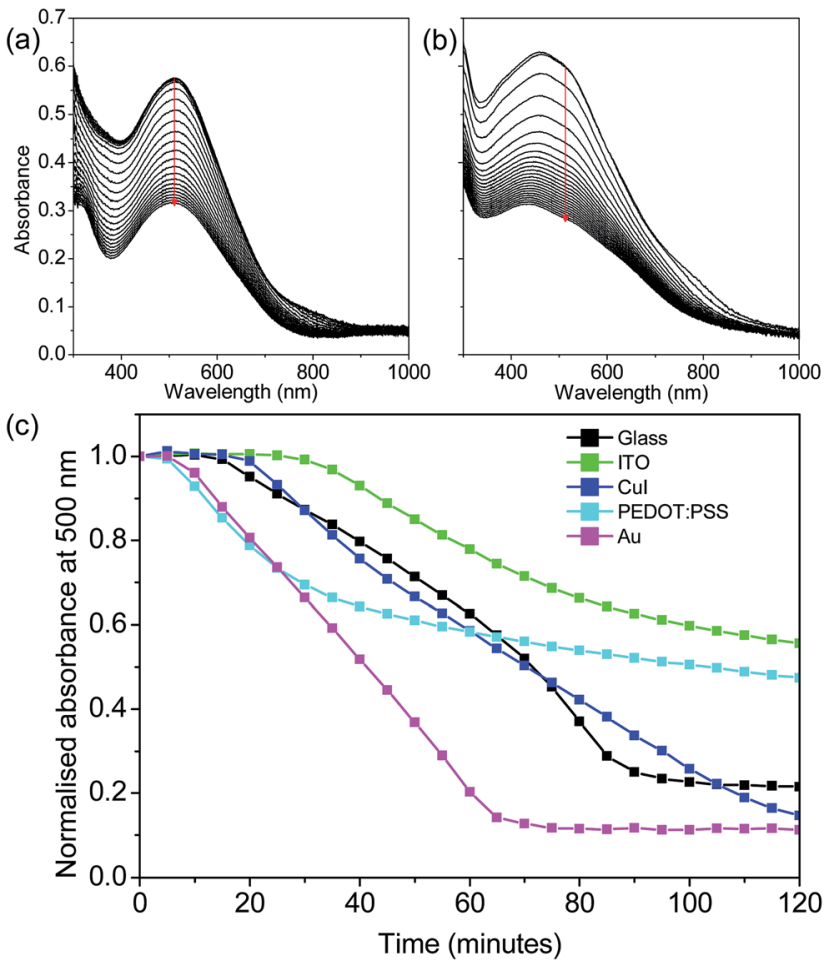

Fig. 1 Evolution of electronic absorption spectrum of $\mathrm{CsSnl}_{3}+$ $10 \mathrm{~mol} \% \mathrm{SnCl}_{2}$ films deposited on ITO glass (a) and PEDOT:PSS (b) when exposed to ambient air $(\mathrm{RH}=38 \%)$. Fig. 1(c) shows the evolution of absorbance at a wavelength of $500 \mathrm{~nm}$ for $\mathrm{CsSnl}_{3}: \mathrm{SnCl}_{2}$ films on ITO, ITO:Cul, ITO:PEDOT:PSS, Au and glass. In all cases the $\mathrm{CsSnl}_{3}$ solution concentration was $8 \mathrm{wt} \%$ which resulted in a film thickness of $\sim 50 \mathrm{~nm}$. In each case the background has been subtracted.

fissures in the film which determines the surface area of film exposed to the environment, and therefore its susceptibility to oxidation; (iii) the intrinsic stability of the underlying substrate material(s) towards oxidation in air; (iv) and the intrinsic stability of the interfaces between the films, and the relative importance (i)-(iv) is not easily disentangled. It is however evident from Fig. 1 and 2 that for perovskite films on ITO glass, glass, CuI and PEDOT:PSS the decrease in perovskite film stability correlates with a decrease in the perovskite coverage of the underlying substrate: excluding the perovskite film on $\mathrm{Au}$, the film on ITO glass is the most compact with a significant number density of pin-holes with diameter 15-25 $\mathrm{nm}$. On CuI the perovskite film has a high density of elongated fissures along grain boundaries, while on the PEDOT:PSS substrate the perovskite film is non-uniform with some regions being densely packed with a pinhole density and pin-hole size comparable to that on ITO glass (Fig. 2(e)), and others have a high density of large fissures (Fig. 2(d)). The decrease in stability with reduced perovskite coverage of the underlying substrate can be rationalised in terms of the greater surface area of the perovskite film presented to the oxidising environment. The stability of the underlying substrate towards ambient air may also play a role given that PEDOT:PSS is known to be hygroscopic ${ }^{39}$ and the first stage of $\mathrm{CsSnI}_{3}$ film oxidation (i.e. conversion of $\mathrm{B}-\gamma \mathrm{CsSnI}_{3}$ to Y-CsSnI ${ }_{3}$ ) results from the interaction with water. ${ }^{17}$ The stability 


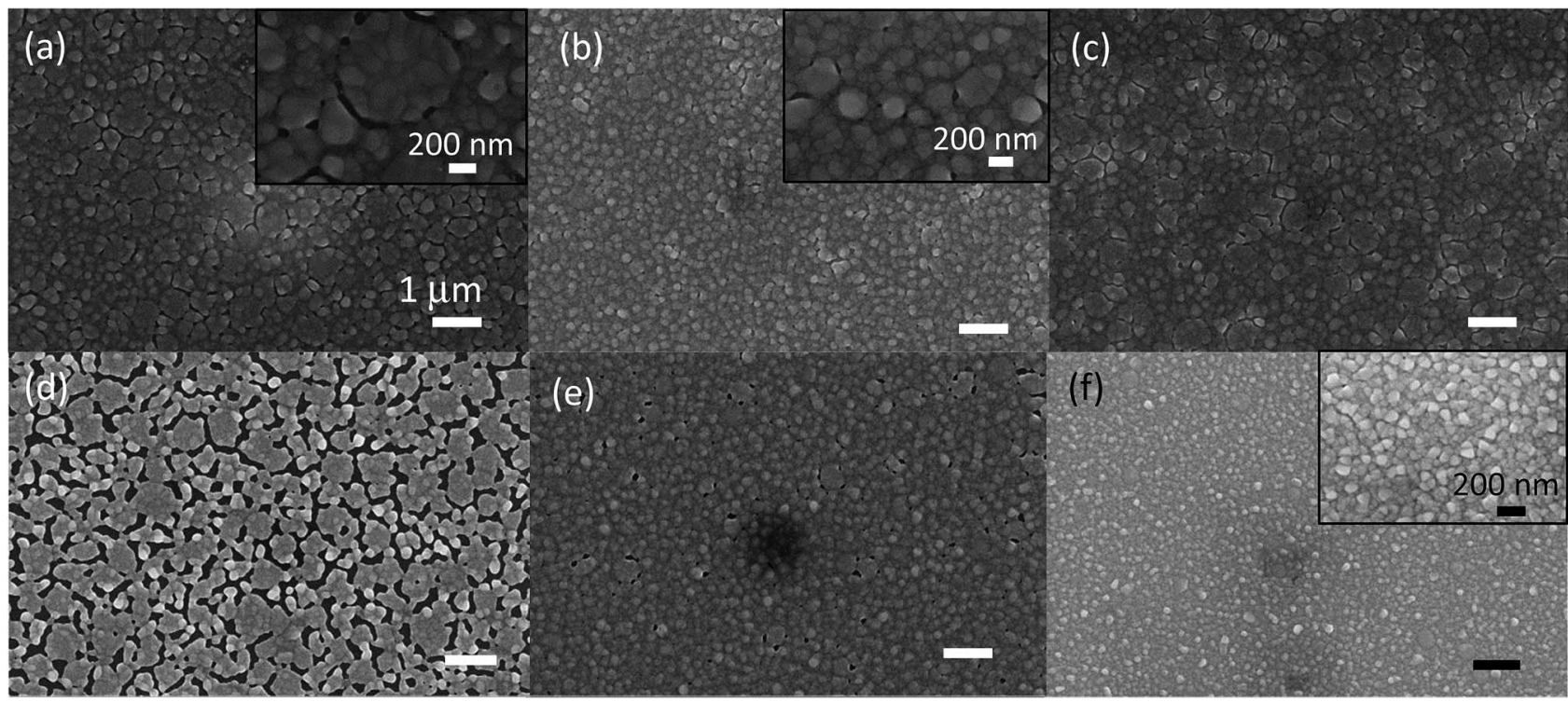

Fig. 2 SEM images of $\mathrm{CsSnl}_{3}+10 \mathrm{~mol}_{\mathrm{SnCl}}$ deposited on top of (a) glass, (b) ITO, (c) Cul, (d) PEDOT:PSS (low coverage section), (e) PEDOT:PSS (high coverage area), (f) Au. The scale bar (b)-(f) is equal to $1 \mu \mathrm{m}$.

of $\mathrm{CsSnI}_{3}: \mathrm{SnCl}_{2}$ film on Au does not follow this trend because it is the least stable towards air-oxidation whilst also has the most compact and uniform film morphology with a relatively low density of very small (13-17 nm) pinholes. Given that the $\mathrm{Au}$ electrode is stable in ambient air, ${ }^{29}$ the poor stability of the $\mathrm{CsSnI}_{3}: \mathrm{SnCl}_{2}$ film on Au most likely stems from instability in the perovskite film itself. Close inspection of the SEM images in Fig. 2(b) and (f) reveals that $\mathrm{CsSnI}_{3}: \mathrm{SnCl}_{2}$ films on $\mathrm{Au}$ have the smallest and most uniform crystallite sizes; $\sim 2200 \mathrm{~nm}^{2} v s$. $\sim 4900 \mathrm{~nm}^{2}$ on ITO glass, and so it is likely that the higher density of grain boundaries enables more rapid water and oxygen penetration into the film, giving rise to increased instability despite the high film coverage of the underlying substrate, as previously been reported for lead halide perovskites. ${ }^{40}$ Collectively these observations show that the stability of $\mathrm{CsSnI}_{3}$ films towards air oxidation depends not only on the density of microscopic pin-holes and fissures but also on the density of grain boundaries between $\mathrm{CsSnI}_{3}$ crystallites, with highest stability offered by perovskite films that are compact and comprise larger crystallites.

X-ray diffraction (XRD) was used to confirm that the $\mathrm{B}-\gamma$ phase of $\mathrm{CsSnI}_{3}$ is formed, and to investigate the possibility that the stability of the perovskite films depends on the orientation of the crystallites making up the film, since different crystal faces would be expected to have different reactivity towards water and oxygen, due to the different arrangement and density of atoms presented to the atmosphere (Fig. 3).

The XRD patterns shown in Fig. 3 confirm the presence of only B- $\gamma \mathrm{CsSnI}_{3}$ on all substrates, and indicate that there is a preferred orientation for crystallites on $\mathrm{CuI}$ and $\mathrm{Au}$, because the intensity of the reflection at $\sim 29^{\circ}$ (202 and 040) is greatly increased as compared to that at $\sim 25^{\circ}$ (220 and 022). However, the large differences in preferred crystallite orientation do not correlate with film stability towards oxidation in air, since films on $\mathrm{CuI}$ and $\mathrm{Au}$ have very similar preferred orientation, but very different stability in air (Fig. 1(c)).

Solution processed $\mathrm{CuI}$ has been reported to be an effective as a HTL in lead perovskite PV prepared using DMF as the solvent for perovskite deposition. ${ }^{23,24}$ However, whilst the SEM image in Fig. 2(c) shows that $\mathrm{CsSnI}_{3}$ films on $\mathrm{CuI}$ have high coverage of the underlying substrate, the XRD pattern; Fig. $\mathrm{S} 4, \dagger$ shows complete disappearance of the intense CuI (111) peak when a $\mathrm{CsSnI}_{3}$ film is deposited on top of a $\mathrm{CuI}$ film by spin coating, indicating the $\sim 40 \mathrm{~nm}$ CuI film is dissolved and displaced by the $\mathrm{CsSnI}_{3}$ solution during the spin coating process. This conclusion is supported by the absorption spectrum of the perovskite film deposited on CuI; Fig. S5, $\dagger$ which shows only a small perturbation of shape of the $\mathrm{CsSnI}_{3}: \mathrm{SnCl}_{2}$ spectrum at wavelengths below $\sim 350 \mathrm{~nm}$, which corresponds to the onset of strong absorption in CuI. Additionally, cross-sectional AFM image analysis of scored $\mathrm{CuI} / \mathrm{CsSnI}_{3}: \mathrm{SnCl}_{2}$ films; Fig. S6, $\uparrow$ shows

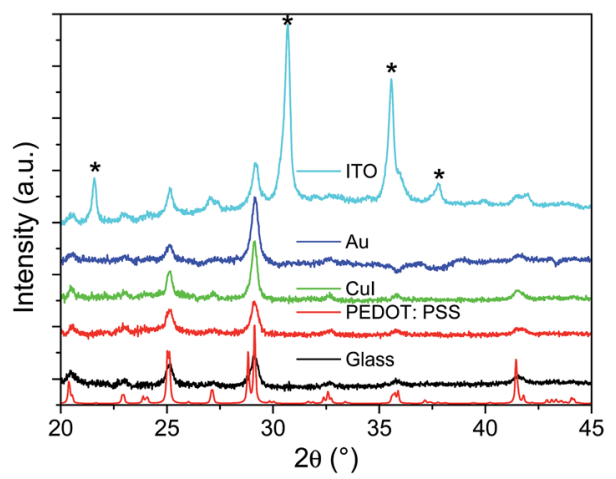

Fig. 3 XRD patterns of films of $\mathrm{CsSnl}_{3}+10 \mathrm{~mol}^{2} \mathrm{SnCl}_{2}$ on ITO, Au, Cul, PEDOT:PSS and glass substrates and simulated. Also shown is the simulated $\mathrm{B}-\gamma \mathrm{CsSnl}_{3}$ spectrum (bottom). Asterisks (*) indicate reflections associated with the ITO background (Fig. S3 †). 
that the measured thickness of the film is $35-40 \mathrm{~nm}$, which is only half the combined thickness of separate CuI and perovskite overlayer. Energy-dispersive X-ray analysis of the films (Fig. S7 and Table $\mathrm{S} 1 \dagger$ ) also show that $\mathrm{Cu}$ is barely detectable in $\mathrm{CuI} / \mathrm{CsSnI}_{3}: \mathrm{SnCl}_{2}$ films supported on ITO glass, whilst the indium signal from the underlying ITO substrate is very intense. AFM imaging and electronic absorption spectroscopy of $\mathrm{CuI}$ films before and after spin casting DMF, Fig. S8(a-c), $\dagger$ reveal that CuI films are partially soluble in DMF, which would be expected to give rise to a complex interpenetrating interface between the CuI and perovskite overlayer, with a significant amount of CuI remaining at the interface between the ITO glass and the perovskite film. However, the weight of experimental evidence is consistent with almost complete displacement of the $\sim 40 \mathrm{~nm}$ CuI film to form a perovskite film with comparable crystallinity and film coverage to that achieved on ITO glass without $\mathrm{Cu}$, which is unexpected given the very rapid speed of the perovskite film formation during spin coating process. It is known that $\mathrm{CuI}$ is soluble in concentrated aqueous solutions of iodide ion, ${ }^{\mathbf{4 1}}$ and so it is plausible that in this case the iodide concentration in the DMF solution used to prepare the perovskite films is sufficiently high to make the solution a very powerful solvent for CuI, which together with the tendency of $\mathrm{CsSnI}_{3}$ to crystallize very rapidly from $\mathrm{DMF}^{42}$ results in the near complete displacement of the CuI film, rendering CuI unsuitable a HTL in inverted $\mathrm{CsSnI}_{3}$ PV devices.

\section{Photovoltaic device studies}

Stability studies under 1 sun continuous illumination in ambient air without device encapsulation were performed on PV devices identical in every respect, except in the choice of hole-extracting electrode and/or HTL, as schematically illustrated in Fig. 4(a).

Ingress of ambient air into a PV device is inevitable even with encapsulation (given sufficient time) and so testing in air under constant illumination is a test condition of practical relevance. However, the degradation mechanisms under such complex conditions are inevitably multi-faceted, with a number of parallel mechanisms operating over different time scales. ${ }^{43}$ For this reason the device structure used to investigate the correlation between device stability and the choice of hole-extracting substrate is based on a simple discrete layer architecture.

It is evident from Fig. 3 and 5 that there is no strong correlation between the preferred crystallite orientation and device stability towards oxidation, since the rate of reduction in absolute efficiency of devices using Au and ITO electrodes is similar, even though a preferred crystallite orientation is much more evident for $\mathrm{CsSnI}_{3}$ films on Au. Consequently, whilst crystallite orientation may play some role in determining $\mathrm{CsSnI}_{3}$ film stability towards air-oxidation, it is much less important than the role of pinholes and grain boundaries between crystallites. For the champion device using ITO only the hole-extracting electrode the efficiency degrades to $70 \%$ of its initial value only after $\sim 20$ hours which is comparable to the highest reported for unencapsulated Sn perovskite based PV devices tested under constant illumination in ambient air. .7,18,31-33,44 $^{-1}$
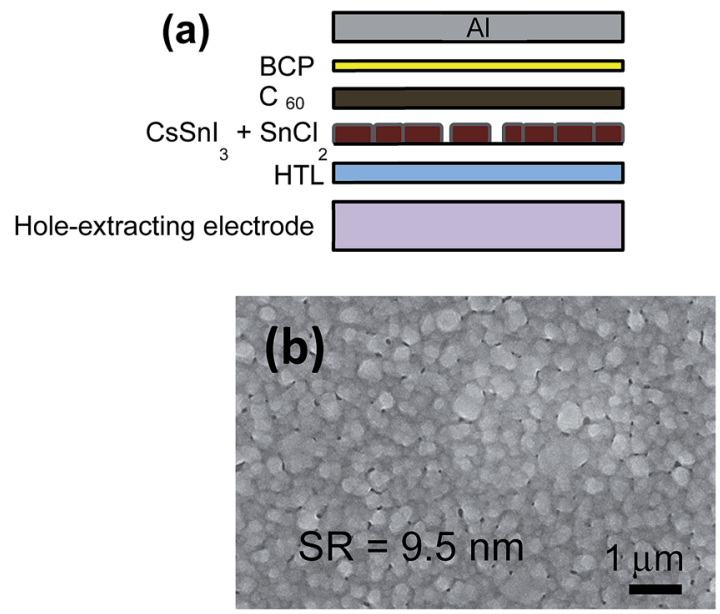

Fig. 4 (a) Schematic diagram of the model device architecture used in this study, including $\mathrm{CsSnl}_{3}$ with pinholes. (b) SEM image of the surface morphology $\mathrm{C}_{60}$ deposited by vacuum evaporation onto a $\sim 50 \mathrm{~nm}$ thick $\mathrm{CsSnl}_{3}$ film supported on ITO glass. The root-mean-square surface roughness (SR) (measured using atomic force microscopy) is given on the image.

The primary reason for the deterioration in the power conversion efficiency of devices using ITO (only) and Au as the hole extracting electrode over the first 24 hours testing in air is the $\sim 50 \%$ loss in short circuit current density $\left(J_{\text {sc }}\right)$, which almost certainly results from several parallel processes some of which will be unrelated to the perovskite layer. For example, it is known that exposure of $\mathrm{C}_{60}$ to ambient air deteriorates its conductivity due to doping by $\mathrm{H}_{2} \mathrm{O}$ and $\mathrm{O}_{2}$, which trap electrons. ${ }^{45,46} \mathrm{BCP}$ is also known to crystallise when exposed to air (a process that will be accelerated by the elevated temperature under the solar simulator; $\sim 50{ }^{\circ} \mathrm{C}$ ) forming electron trap states at the interface between crystallites. ${ }^{47}$ Air ingress into the device through pin holes in the $\mathrm{Al}$ electrode is also known to result in the formation of an insulating $\mathrm{Al}_{2} \mathrm{O}_{3}$ layer at the in $\mathrm{BCP} / \mathrm{Al}$ interface. ${ }^{4-50}$ Any of these processes could give rise to the observed increase in device series resistance, which is evident from the decrease in gradient of the $J-V$ characteristic where it crosses the voltage axis: Fig. S9(a)-(c). $\dagger$

Assuming the efficiency of hole-extraction across the buried interface between the ITO (or Au) electrode and perovskite remains unchanged, any reduction in the efficiency of electron extraction to the external circuit as a result of any of the aforementioned degradation processes would be expected to increase recombination losses in the perovskite layer, offering a plausible explanation for the observed decline in $J_{\mathrm{sc}}{ }^{51}$ However, air ingress into the device would also be expected result in oxidation of the $\mathrm{CsSnI}_{3}$ to form $\mathrm{Cs}_{2} \mathrm{SnI}_{6},{ }^{17}$ resulting in two additional mechanisms for $J_{\text {sc }}$ degradation: (i) $\mathrm{Cs}_{2} \mathrm{SnI}_{6}$ is a semiconductor with an absorption coefficient across the visible spectrum $\sim 10 \times$ smaller than that of $\mathrm{B}-\gamma \mathrm{CsSnI}_{3}$, and so the transformation of $\mathrm{CsSnI}_{3}$ into $\mathrm{Cs}_{2} \mathrm{SnI}_{6}$ would reduce the light harvesting capability of the device, thereby reducing $J_{\text {sc }}$; (ii) the work function and energy of the valence band edge of $\mathrm{Cs}_{2} \mathrm{SnI}_{6}$ (prepared by air oxidation of $\mathrm{CsSnI}_{3}$ ) measured using ultra-violet photo-electron spectroscopy (UPS) as part of this 

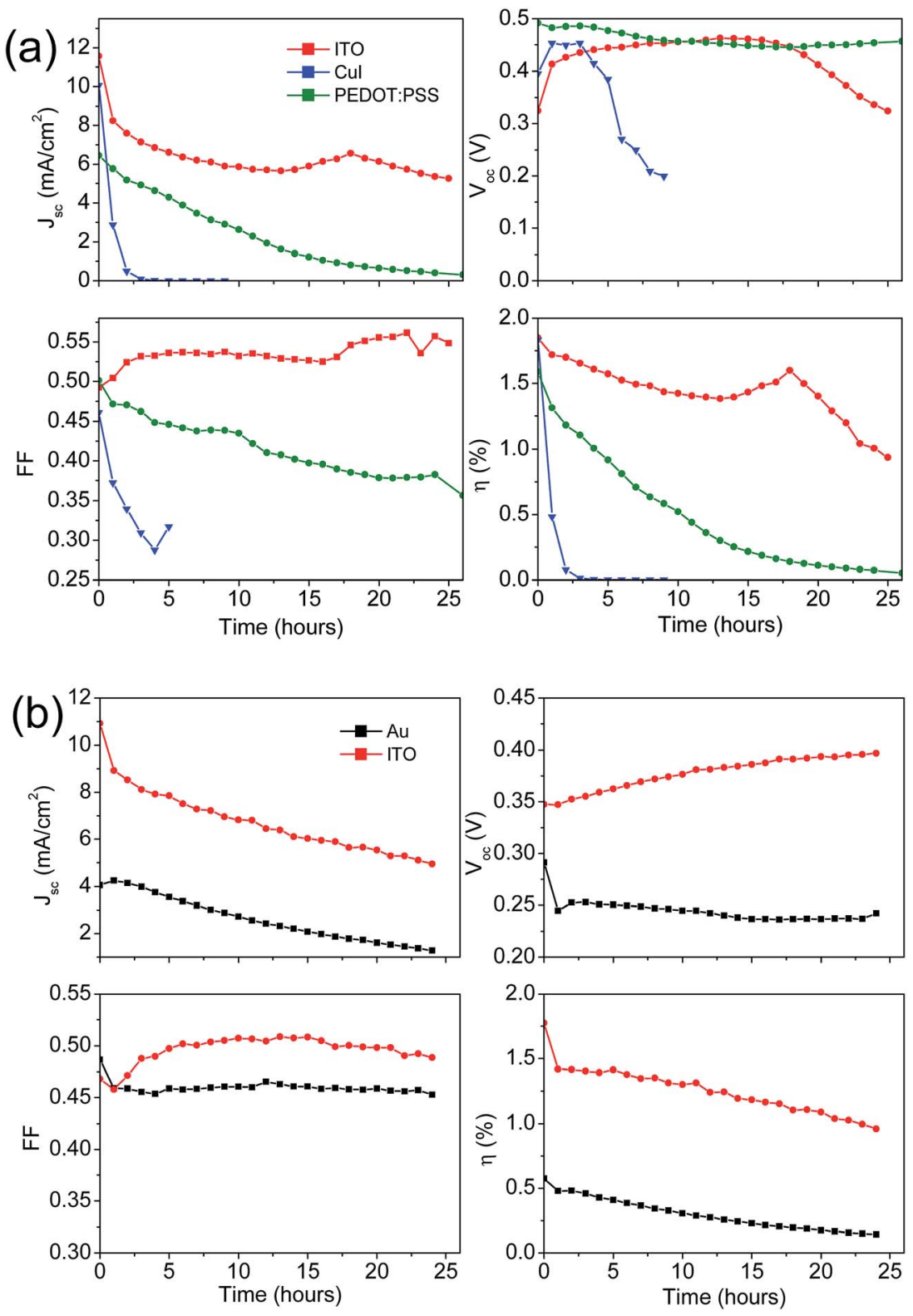

Fig. 5 Representative performance of $6 \mathrm{~mm}^{2} \mathrm{CsSnl}_{3}: \mathrm{SnCl}_{2}$ PPV devices with the structure shown in Fig. 4(a) and without device encapsulation, tested in ambient air (humidity of $\sim 25 \%$ ) under constant 1 sun simulated solar illumination. After 45 minutes the device temperature had stabilised at $\sim 50^{\circ} \mathrm{C}$. The devices tested were identical in every respect except for the choice of hole-extracting electrode/HTL: ITO (a \& b), PEDOT:PSS (a), $\mathrm{Cul}$ (a), or $\mathrm{Au}$ (b) substrates. The performance of devices using ITO only, tested in a nitrogen atmosphere $\left(<1 \mathrm{ppm} \mathrm{O}_{2},<1 \mathrm{ppm} \mathrm{H}_{2} \mathrm{O}\right)$ is given in the ESI Fig. 10.†

study with special care to remove adsorbed water and carbon contaminants; Fig. S11, $\uparrow$ are $5.1 \mathrm{eV}$ and $5.83 \mathrm{eV}$ below the vacuum level, respectively. Given that the band gap of $\mathrm{Cs}_{2} \mathrm{SnI}_{6}$ has been reported to be $\sim 1.3 \mathrm{eV}$ (ref. 11 and ${ }^{52}$ ) and $\sim 1.6 \mathrm{eV},{ }^{53}$ the conduction band edge in $\mathrm{Cs}_{2} \mathrm{SnI}_{6}$ is estimated to be in the range 4.2-4.5 eV below the vacuum level (Fig. 6). Whilst both of these limits are well below the energy of the conduction band edge in $\mathrm{CsSnI}_{3} ; 3.6 \mathrm{eV},{ }^{17}$ and the lowest unoccupied molecular orbital energy of $\mathrm{C}_{60} ; 4.0 \mathrm{eV},{ }^{54}$ at the real interface between $\mathrm{CsSnI}_{3}$ and $\mathrm{Cs}_{2} \mathrm{SnI}_{6}$ electron transfer from the $\mathrm{CsSnI}_{3}$ into $\mathrm{Cs}_{2} \mathrm{SnI}_{6}$ would be expected to occur, driven by the differences in Fermi level energy, which would reduce the barrier to electron transport across this interface possibly to $<0.1 \mathrm{eV}$. There is also a large degree of uncertainty as to the extent of charge transfer at the $\mathrm{Cs}_{2} \mathrm{SnI}_{6} / \mathrm{C}_{60}$ interface, which will depend on the local $\mathrm{SnCl}_{2}$ doping level in the $\mathrm{C}_{60}$ layer. Consequently it is not possible to know with a high degree of accuracy the extent to which the formation of $\mathrm{Cs}_{2} \mathrm{SnI}_{3}$ at the interface between $\mathrm{CsSnI}_{3}$ and $\mathrm{C}_{60}$ would impede the flow of electrons to the external circuit, giving rise to an increase in device series resistance. However, it can be concluded that $\mathrm{Cs}_{2} \mathrm{SnI}_{6}$ formed at the $\mathrm{CsSnI}_{3} /$ $\mathrm{C}_{60}$ interface due to air ingress into the device would serve as reservoir of trapped electrons occupying energy states with energies in the band gap of the $\mathrm{CsSnI}_{3}$, the presence of which 

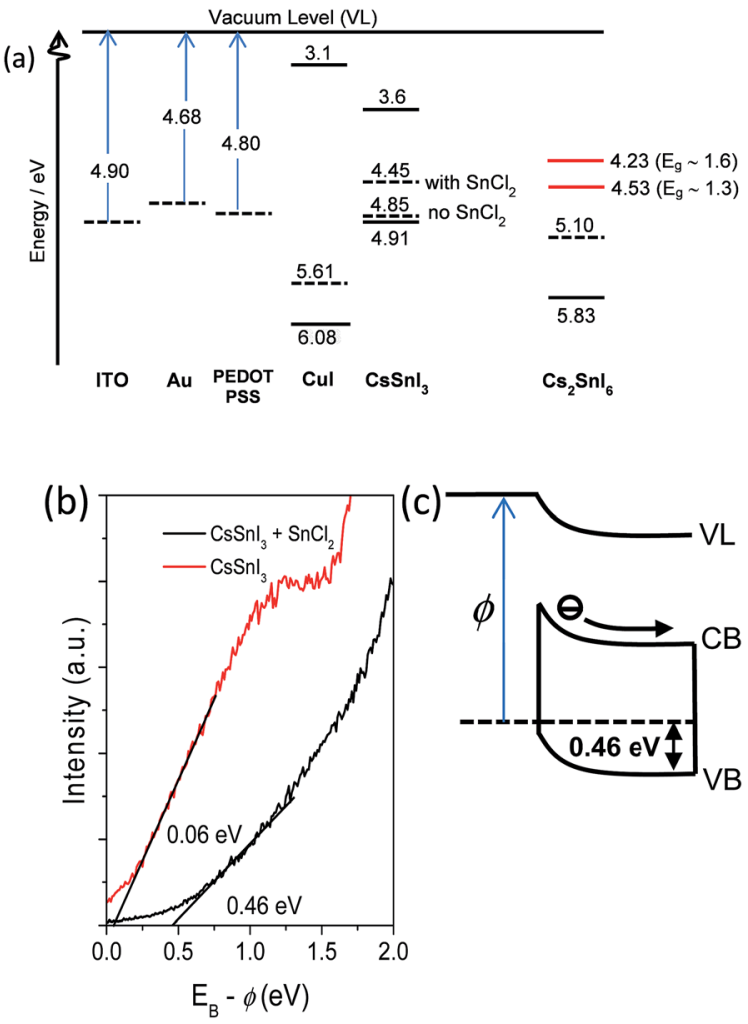

Fig. 6 (a) Schematic energy level diagram summarizing the energy of the valence band (VB) edge with respect to the vacuum level (VL), the work function $(\phi)$ and Fermi level $\left(E_{f}\right)$ (dotted line) for each material used in this study. (b) UPS spectrum of $\mathrm{CsSnl}_{3}$ and $\mathrm{CsSnl}_{3}: \mathrm{SnCl}_{2}$ at VB band edge. The small signal above $E_{\mathrm{f}}$ (i.e. $E_{\mathrm{B}}-\phi>0 \mathrm{eV}$ ) results from $\sim 1 \%$ of the incident UV photons from the UV lamp having energy $>21.22 \mathrm{eV}$. (c) Energy level diagram depicting the Schottky contact between the $\mathrm{CsSnl}_{3}: \mathrm{SnCl}_{2}$ and the ITO electrode.

would be expected to erode $J_{\text {sc }}$ due to increased recombination loses.

To determine the extent of formation of $\mathrm{Cs}_{2} \mathrm{SnI}_{6}$ in an actual devices, devices with a cell area of $\sim 74 \mathrm{~mm}^{2}$ were fabricated and tested under continuous 1 sun simulated illumination for 24 hours: Fig. S12. $\dagger$ The evolution of $V_{\mathrm{oc}}, \mathrm{FF}$ and $J_{\mathrm{sc}}$ of these larger cell area devices is very similar to that for much smaller area $\left(6 \mathrm{~mm}^{2}\right)$ devices shown in Fig. 5 , and is discussed in detail in the next part of this paper. Following device stability testing the $\mathrm{C}_{60}$, BCP and Al layers were removed by peeling off the $\mathrm{Al}$ electrode followed by repeated washing with chlorobenzene, and the absorption spectrum of the remaining $\mathrm{CsSnI}_{3}$ measured to determine the extent of film oxidation: Fig. S13(a). $\dagger$ Most strikingly, the rate of degradation of light absorption by the perovskite film after 24 hours testing is much slower than the rate of degradation in $J_{\mathrm{sc}}$ : after 24 hours $J_{\mathrm{sc}}$ has decreased by $\sim 75 \%$ ( $\sim 50 \%$ in $6 \mathrm{~mm}^{2}$ devices) whilst the absorption intensity (@500 $\mathrm{nm}$ ) is reduced only by $0-11 \%$ over the same time period (Fig. S12†). Quantification of the reduction in absorption intensity with a higher degree of certainty is not possible because the variation in absorbance for $\mathrm{CsSnI}_{3}$ films prepared in the same way is comparable to the reduction in absorbance intensity for films in devices after 24 hours testing; Fig. S13(b). $\dagger$
High resolution XPS analysis of the perovskite film following removal of the $\mathrm{C}_{60}, \mathrm{BCP}$ and $\mathrm{Al}$ layers after 24 hours continuous illumination testing gives the thickness of the $\mathrm{Cs}_{2} \mathrm{SnI}_{6}$ layer to be $\sim 3 \%$ (or $\sim 1.5 \mathrm{~nm}$ ): Fig. S14. $\dagger$ Whilst there is uncertainty associated with this value, due to the presence of a small but significant $\mathrm{Cl} 2 \mathrm{p}$ peak in the XPS spectrum (Fig. S14 lower†) which indicates the presence of residual $\mathrm{SnCl}_{2}$, it is well within the range determined from the electron absorption spectroscopy. This finding indicates that the large deterioration in $J_{\mathrm{sc}}-$ which is the primary reason for the degradation in device efficiency - does not primarily result from a reduction in the light absorption capability of the perovskite film due to oxidation of the $\mathrm{CsSnI}_{3}$ layer. Indeed, for a tin perovskite PV, such a low level of perovskite oxidation after 24 hours continuous illumination in air without encapsulation is remarkable.

The most striking conclusion from a comparison of the device performance (Fig. 5) is that devices with an ITO or Au electrode without a HTL have superior stability to those using the archetypal HTL layer PEDOT:PSS. The high stability of devices using the Au electrode is also counter to that expected on the basis that the $\mathrm{CsSnI}_{3}$ film stability measurements (Fig. 1). To rationalise the latter, it is necessary to take into account not only the intrinsic stability of each semiconductor layer making up the device but also their combined morphology, since the morphology and uniformity of the Al top electrode is strongly dependant on that of the underlying materials. Since the Al electrode is the primary barrier to the ingress of water and oxygen in these devices, ${ }^{50,55}$ any fine gaps or pinholes in the $\mathrm{Al}$ electrode resulting from the high surface roughness of the semiconductor layers onto which it is deposited will have a major adverse effect on device stability. ${ }^{56,57}$ In this work all of the organic semiconductor layers are thermally evaporated to guarantee a high degree of control and reproducibility film thickness. However, unlike solution processed organic semiconductors, evaporated organic semiconductor layers tend to have a morphology very similar to that of the substrate onto which they are deposited, and so the pinholes in the $\mathrm{CsSnI}_{3}: \mathrm{SnCl}_{2}$ film are also present in the $\mathrm{C}_{60}$ over layer (Fig. S15†). Consequently, organic semiconductor films vacuum deposited onto a very uniform and compact perovskite film, such as that formed on Au (Fig. 2(f)), can be expected to have a reduced number of defects in the $\mathrm{C}_{60}$, $\mathrm{BCP}$ and $\mathrm{Al}$ layers that may allow $\mathrm{H}_{2} \mathrm{O}$ and $\mathrm{O}_{2}$ ingress into the device.

The simplest explanation for the inferior device stability using PEDOT:PSS/ITO glass electrode is that the low surface coverage in many areas (Fig. 2(d)) and its well-known hygroscopic properties. ${ }^{58}$ The latter is particularly problematic for $\mathrm{CsSnI}_{3}$ because it is known to degrade in the presence of water to form $\mathrm{Y}_{-} \mathrm{CsSnI}_{3}$, or $\mathrm{Cs}_{2} \mathrm{SnI}_{6}$ when oxygen is also present. ${ }^{9,13}$ However, it is also notable that the rate of degradation in efficiency is much more pronounced than on ITO because the FF and $V_{\text {oc }}$ do not initially improve, as is observed to be the case for devices using ITO without a PEDOT:PSS layer. The initial improvement in these parameters for devices using ITO without a HTL occurs over several hours under constant illumination and is similar to that previously reported to occur after a period of extended storage in a nitrogen filled glovebox for devices 
using $\mathrm{PC}_{61} \mathrm{BM}$ in place of evaporated $\mathrm{C}_{60} \cdot{ }^{17}$ The latter was shown to result from n-type doping of the $\mathrm{PC}_{61} \mathrm{BM}$ by the $\mathrm{SnCl}_{2}$, which results in the formation of a Schottky barrier to parasitic electron extraction by the ITO electrode at the site of small pinholes in the perovskite film. ${ }^{17}$ Direct evidence for an n-type doping interaction between evaporated $\mathrm{C}_{60}$ and $\mathrm{SnCl}_{2}$ is provided by high resolution X-ray photo-electron spectroscopy (Fig. S16 and $\mathrm{S} 17 \dagger)$, which shows that the binding energies of the $\mathrm{Cl} 2 \mathrm{p}$ electrons in $\mathrm{SnCl}_{2}$ incorporated into a $\mathrm{C}_{60}$ film are 198.8$199.1 \mathrm{eV}\left(2 \mathrm{p}_{3 / 2}\right)$ and $200.5-200.8 \mathrm{eV}\left(2 \mathrm{p}_{1 / 2}\right)$, which are $\sim 0.6 \mathrm{eV}$ higher than that of $\mathrm{SnCl}_{2} ; 198.2 \mathrm{eV}$ and $199.8 \mathrm{eV}$ respectively. ${ }^{17}$ This large chemical shift to higher binding energy is consistent with partial electron transfer from the $\mathrm{SnCl}_{2}$ into the $\mathrm{C}_{60}$, similar to that reported to occur between $\mathrm{SnCl}_{2}$ and PCBM. ${ }^{17}$ It is reasonable to expect that diffusion of $\mathrm{SnCl}_{2}$ into $\mathrm{C}_{60}$ is accelerated by the heating under the solar simulator lamp, since after 45 minutes exposure to one sun simulated solar illumination the devices stabilise at a temperature of $\sim 50{ }^{\circ} \mathrm{C}$, and so $\mathrm{C}_{60}$ at the site of pin-holes in the $\mathrm{CsSnI}_{3}$ film takes time to become optimally doped with $\mathrm{SnCl}_{2}$.

The dark current-voltage characteristics of the same devices before and after five hours continuous illumination plotted on a log-linear scale (Fig. S18†) validate the conclusion that a barrier to parasitic electron extraction forms at the ITO electrode, since the dark current in reverse bias is dramatically reduced. Within the framework of this model the lack of improvement in $V_{\text {oc }}$ and FF for devices using PEDOT:PSS can be understood in terms of the very large size of the holes in $\mathrm{CsSnI}_{3}: \mathrm{SnCl}_{2}$ layer on PEDOT:PSS (Fig. 1(d)), because the $\mathrm{SnCl}_{2}$ concentration in the $\mathrm{C}_{60}$ at the site of these large holes is unlikely to be as high as at the site of the much smaller pinholes in the $\mathrm{CsSnI}_{3}: \mathrm{SnCl}_{2}$ layer on ITO (Fig. 1(b)) where $\mathrm{SnCl}_{2}$ on the side walls of the $\mathrm{CsSnI}_{3}$ crystallites will also be a significant source of $\mathrm{SnCl}_{2}$. Conversely, the absence of any improvement in $V_{\mathrm{oc}}$ and FF for devices using an Au hole-extracting electrode can be explained by the very compact, almost pin-hole free morphology of the perovskite film on Au (Fig. 2(f)), which means that the aforementioned mechanism of improvement in FF and $V_{\text {oc }}$ would not be operative.

CuI has been proposed as an attractive alternative to PEDOT:PSS for lead perovskite PV devices due to its wide band gap, and ease with which it can be solution processed to form thin films and high stability. ${ }^{23-25}$ However, it is evidence from Fig. 6 that devices using CuI as the HTL are in fact very unstable, exhibiting a rapid deterioration in $\mathrm{FF}$ and $J_{\mathrm{sc}}$ when tested under continuous illumination. This instability is attributed to the previously discussed disordered nature and very low thickness of the CuI layer at the ITO/perovskite interface after perovskite deposition. Measurement of the work function and energy of the valence band edge with respect the vacuum level (Fig. S19 \& $20 \dagger$ ), schematically shown in Fig. 6, reveals that whilst the valence band in $\mathrm{CsSnI}_{3}$ should be closely aligned with the valence band in CuI once thermodynamic equilibrium is established, holes could become trapped in the potential well at the interface between them, which would be expected to reduce $J_{\mathrm{sc}}$ by reducing the built-in electric field. However, the $J_{\mathrm{sc}}$ is in fact comparable to that using ITO with no HTL, which is consistent with the finding that the CuI layer is almost completely displaced by the perovskite film during $\mathrm{CsSnI}_{3}$ film deposition.

Analysis of the valence band edge region in the UPS spectrum (Fig. 5(b)) shows that the difference in energy between the Fermi level $\left(E_{\mathrm{f}}\right)$ and the valence band in $\mathrm{CsSnI}_{3}$ is increased by $\sim 0.4 \mathrm{eV}$ when $\mathrm{CsSnI}_{3}$ is synthesised in the presence of $10 \mathrm{~mol} \%$ $\mathrm{SnCl}_{2}$. This large shift away from the valence band confirms that $\mathrm{SnCl}_{2}$ serves to reduce the density of Sn vacancy defects, which are known to be the primary source of background carrier density in $\mathrm{CsSnI}_{3} .{ }^{14}$ It can be surmised from Fig. 6 that there should be no barrier to the extraction of photo-generated holes using ITO, Au or ITO| PEDOT:PSS since the work function $(\phi)$ of $\mathrm{CsSnI}_{3}: \mathrm{SnCl}_{2}$ is smaller than all of the hole-extraction electrodes/HTL materials investigated, and the valence band edge in $\mathrm{CsSnI}_{3}: \mathrm{SnCl}_{2}$ is lower in energy than the Fermi level in ITO, Au or PEDOT:PSS/ITO. Consequently, the energetics upon contact formation should facilitate hole-extraction, as schematically illustrated in Fig. 6(c). Notably, however, devices using ITO|PEDOT:PSS initially exhibit approximately half the $J_{\mathrm{sc}}$ of those using ITO only. Optical modelling of the optical field distribution in the PEDOT:PSS shows that this large difference in $J_{\text {sc }}$ cannot be attribute to a difference in light intensity in the $\mathrm{CsSnI}_{3}$ layer, which is comparable for devices with and without PEDOT:PSS (Fig. S21(a) and (b) $\dagger$ ). The difference in $J_{\text {sc }}$ can however be explained in terms of the difference in the $\phi$ of the PEDOT:PSS and ITO glass, because the former is $\sim 0.1 \mathrm{eV}$ smaller than that of ITO. Whilst the absolute magnitude of this difference it relatively small, it is significant compared to the difference in energy between the $E_{\mathrm{f}}$ in $\mathrm{CsSnI}_{3}: \mathrm{SnCl}_{2}$ and the hole-extracting electrode $E_{\mathrm{f}}(0.35-0.45 \mathrm{eV})$, which would result in a narrower depletion region in the $\mathrm{CsSnI}_{3}$ when using PEDOT:PSS as the electrode. It is over the depletion region that photo-generated electrons and holes are most efficiently separated in a perovskite $\mathrm{PV}$ device, ${ }^{59}$ and so reducing the width of the depletion region would be expected to reduce $J_{\mathrm{sc}}-$ as is observed to be the case. This rationale also offers a plausible explanation for the low $J_{\mathrm{sc}}$ in devices using an optically thin $\mathrm{Au}$ electrode, which have a $J_{\text {sc }}$ only one third of that achieved using an ITO electrode, even though the far-field transparency of the $\mathrm{Au}$ electrode is approximately two thirds that of ITO glass. ${ }^{29}$ Again, optical modelling confirms that the low $J_{\mathrm{sc}}$ cannot be explained in terms of the lower transparency of the Au electrode alone: Fig. S21(a) and (c). $\dagger$ However, the $\phi$ of the Au electrode is significantly smaller than that of ITO or PEDOT:PSS electrode, which would further reduce the depletion width, and thus the perovskite thickness over which photo-generated charge carriers are efficiency separated.

\section{Conclusions}

The key findings of this study are:

(1) Using five different types of substrate we have shown that the stability of thin films of $\mathrm{B}-\gamma \mathrm{CsSnI}_{3}$ perovskite towards oxidation in air depends strongly not only on the density of microscopic pinholes and fissures, but also on the density of grain boundaries between $\mathrm{CsSnI}_{3}$ crystallites, with best stability 
offered by films that are compact and comprise of larger crystallites. X-ray diffraction measurements performed on perovskite films prepared in the same way as for PV devices, show that the stability of $\mathrm{CsSnI}_{3}$ films towards oxidation in air is not strongly dependent on preferred orientation of the $\mathrm{CsSnI}_{3}$ crystallites.

(2) We have shown that CuI is unsuitable as the HTL in $\mathrm{CsSnI}_{3}$ inverted PV when using DMF as the solvent, because it is almost completely displaced by the $\mathrm{CsSnI}_{3}$ precursor solution during the spin coating process and its large ionisation potential is poorly matched to the valence band edge of $\mathrm{CsSnI}_{3}$. It is likely that a similar problem will arise if DMF is substituted with an alternative polar solvent.

(3) It is shown that unencapsulated PV devices based on an inverted bilayer device architecture using ITO or semitransparent $\mathrm{Au}$, as the hole-extracting electrode without a HTL are more stable than those using the archetypal hole-extraction material PEDOT:PSS when tested under 1 sun constant simulated illumination in ambient air. This difference is attributed to the inferior film forming properties of $\mathrm{CsSnI}_{3}$ on PEDOT:PSS combined with its well-known hygroscopic nature. Whilst chemical modification of PEDOT:PSS to rectify the problem of film uniformity may be possible, the hygroscopic nature is a particular problem for $\mathrm{CsSnI}_{3}$ based PVs because $\mathrm{H}_{2} \mathrm{O}$ in known to initiate oxidation of $\mathrm{CsSnI}_{3}$, and so hygroscopic materials immediately adjacent to $\mathrm{CsSnI}_{3}$ should be avoided.

(4) PV devices using ITO only as the hole-extracting electrode exhibit the highest stability, with $30 \%$ reduction in efficiency only after $\sim 20$ hours testing in air for the champion device, which is very high for an unencapsulated tin perovskite PV device test in air. However, devices using a model semitransparent $8 \mathrm{~nm}$ gold window electrode also exhibit good stability, which indicates that this simplification in device structure could be generalised to other stable hole-extracting electrodes.

(5) The reason for the deterioration in devices using ITO, Au and PEDOT:PSS with time is primarily degradation in $J_{\mathrm{sc}}$. However, analysis of the composition of the upper surface of the $\mathrm{CsSnI}_{3}$ layer recovered from devices tested in ambient air for 24 hours without device encapsulation, reveals that $\leq 11 \%$ of the $\mathrm{CsSnI}_{3}$ film has oxidised to $\mathrm{Cs}_{2} \mathrm{SnI}_{6}$. Consequently, the deterioration in device efficiency over this time frame does not primarily result from a reduction in the light absorption capability of the perovskite film due to oxidation of the $\mathrm{CsSnI}_{3}$ layer.

(6) Finally, the results of the photoelectron spectroscopy study has shown (for the first time) that when $\mathrm{CsSnI}_{3}$ is synthesised in the presence of $\mathrm{SnCl}_{2}$ as a source of excess $\mathrm{Sn}$ the work function of the perovskite is reduced from $\sim 4.85 \mathrm{eV}$ to $\sim 4.45 \mathrm{eV}$, which is consistent with a reduction in the density of Sn vacancy defects in the perovskite. Notably, this reduction in work function ensures that the work function of $\mathrm{CsSnI}_{3}$ is smaller than most hole-extracting electrode materials used in perovskite and organic PV research, and so in most instances a Schottky contact will be formed that would operate to selectively block the unwanted extraction of electrons. This, combined with the added complexity in fabrication and potential for additional degradation pathways that comes with the inclusion of a HTL into the device architecture, brings into question the rationale for the inclusion of HTL in this type of device.

\section{Conflicts of interest}

There are no conflicts to declare.

\section{Acknowledgements}

The authors would like to thank the United Kingdom Engineering and Physical Sciences Research Council (EPSRC) for funding (Grant number: EP/L505110/1 \& EP/N009096/1), and Philip Bellchambers for preparing a sample for photoelectron spectroscopy. All data supporting this study are provided as supplementary information accompanying this paper.

\section{References}

1 A. Kojima, K. Teshima, Y. Shirai and T. Miyasaka, J. Am. Chem. Soc., 2009, 131, 6050.

2 N. K. Elumalai, M. A. Mahmud, D. Wang and A. Uddin, Energies, 2016, 9, 861.

3 M. Li, H. Gou, I. Al-Ogaidi and N. Wu, ACS Sustainable Chem. Eng., 2013, 1, 713.

4 G. Flora, D. Gupta and A. Tiwari, Interdiscip. Toxicol., 2012, 5, 47.

5 F. Giustino and H. J. Snaith, ACS Energy Lett., 2016, 1, 1233. 6 European Food Safety Authority, EFSA J., 2010, 8, 1570.

7 W. Huang, J. S. Manser, P. V. Kamat and S. Ptasinska, Chem. Mater., 2016, 28, 303.

8 A. Babayigit, A. Ethirajan, M. Muller and B. Conings, Nat. Mater., 2016, 15, 247.

9 I. Chung, J.-H. Song, J. Im, J. Androulakis, C. D. Malliakas, H. Li, A. J. Freeman, J. T. Kenney and M. G. Kanatzidis, J. Am. Chem. Soc., 2012, 134, 8579.

10 Z. Chen, C. Yu, K. Shum, J. J. Wang, W. Pfenninger, N. Vockic, J. Midgley and J. T. Kenney, J. Lumin., 2012, 132, 345.

11 J. Zhang, C. Yu, L. Wang, Y. Li, Y. Ren and K. Shum, Sci. Rep., 2014, 4, 6954.

12 M. H. Kumar, S. Dharani, W. L. Leong, P. P. Boix, R. R. Prabhakar, T. Baikie, C. Shi, H. Ding, R. Ramesh, M. Asta, M. Graetzel, S. G. Mhaisalkar and N. Mathews, Adv. Mater., 2014, 26, 7122.

13 C. C. Stoumpos, C. D. Malliakas and M. G. Kanatzidis, Inorg. Chem., 2013, 52, 9019.

14 P. Xu, S. Chen, H.-J. Xiang, X.-G. Gong and S.-H. Wei, Chem. Mater., 2014, 26, 6068.

15 K. P. Marshall, R. I. Walton and R. A. Hatton, J. Mater. Chem. A, 2015, 3, 11631.

16 T. M. Koh, T. Krishnamoorthy, N. Yantara, C. Shi, W. L. Leong, P. P. Boix, A. C. Grimsdale, S. G. Mhaisalkar and N. Mathews, J. Mater. Chem. A., 2015, 3, 14996.

17 K. P. Marshall, M. Walker, R. I. Walton and R. A. Hatton, Nat. Energy, 2016, 1, 16178. 
18 S. Gupta, T. Bendikov, G. Hodes and D. Cahen, ACS Energy Lett., 2016, 1, 1028.

19 Food Standards Agency, Current EU approved additives and their E Numbers, 2016, http://www.food.gov.uk/science/additives/ enumberlist.

20 Y. Zhang, X. Hu, L. Chen, Z. Huang, Q. Fu, Y. Liu, L. Zhang and Y. Chen, Org. Electron., 2016, 30, 281.

21 A. Mei, X. Li, L. Liu, Z. Ku, T. Liu, Y. Rong, M. Xu, M. Hu, J. Chen, Y. Yang, M. Grätzel and H. Han, Science, 2014, 345, 295.

22 L. Meng, J. You, T.-F. Guo and Y. Yang, Acc. Chem. Res., 2016, 49, 155.

23 W.-Y. Chen, L.-L. Deng, S.-M. Dai, X. Wang, C.-B. Tian, X.-X. Zhan, S.-Y. Xie, R.-B. Huang and L. Zheng, J. Mater. Chem. A, 2015, 3, 19353.

24 W. Sun, S. Ye, H. Rao, Y. Li, Z. Liu, L. Xiao, Z. Chen, Z. Bian and C. Huang, Nanoscale, 2016, 8, 15954.

25 J. A. Christians, R. C. M. Fung and P. V. Kamat, J. Am. Chem. Soc., 2014, 136, 758.

26 H.-B. Kim, H. Choi, J. Jeong, S. Kim, B. Walker, S. Song and J. Y. Kim, Nanoscale, 2014, 6, 6679.

27 X. Bao, Y. Wang, Q. Zhu, N. Wang, D. Zhu, J. Wang, A. Yang and R. Yang, J. Power Sources, 2015, 297, 53.

28 J.-Y. Jeng, Y.-F. Chiang, M.-H. Lee, S.-R. Peng, T.-F. Guo, P. Chen and T.-C. Wen, Adv. Mater., 2013, 25, 3727.

29 H. M. Stec, R. J. Williams, T. S. Jones and R. A. Hatton, Adv. Funct. Mater., 2011, 21, 1709.

30 H. M. Stec and R. A. Hatton, ACS Appl. Mater. Interfaces, 2012, 4, 6013.

31 D. Moghe, L. Wang, C. J. Traverse, A. Redoute, M. Sponseller, P. R. Brown, V. Bulović and R. R. Lunt, Nano Energy, 2016, 28, 469.

32 W. Liao, D. Zhao, Y. Yu, C. R. Grice, C. Wang, A. J. Cimaroli, P. Schulz, W. Meng, K. Zhu, R. G. Xiong and Y. Yan, Adv. Mater., 2016, 28, 9333.

33 F. Hao, C. C. Stoumpos, D. H. Cao, R. P. H. Chang and M. G. Kanatzidis, Nat. Photonics, 2014, 8, 489.

34 Thin Film Center Inc., 2013.

35 C. F. Macrae, I. J. Bruno, J. A. Chisholm, P. R. Edgington, P. McCabe, E. Pidcock, L. Rodriguez-Monge, R. Taylor, J. Van De Streek and P. A. Wood, J. Appl. Crystallogr., 2008, 41, 466.

36 N. Fairley, CasaXPS, Casa Software Ltd., 2016.

37 I. Horcas, R. Fernández, J. M. Gómez-Rodríguez, J. Colchero, J. Gómez-Herrero and A. M. Baro, Rev. Sci. Instrum., 2007, 78, 13705.

38 C. A. Schneider, W. S. Rasband and K. W. Eliceiri, Nat. Methods, 2012, 9, 671.

39 J. Zhou, D. H. Anjum, L. Chen, X. Xu, I. A. Ventura, L. Jiang and G. Lubineau, J. Mater. Chem. C, 2014, 2, 9903.

40 A. M. A. Leguy, Y. Hu, M. Campoy-Quiles, M. I. Alonso, O. J. Weber, P. Azarhoosh, M. van Schilfgaarde,
M. T. Weller, T. Bein, J. Nelson, P. Docampo and P. R. F. Barnes, Chem. Mater., 2015, 27, 3397.

41 G. B. Kauffman, L. Y. Fang, N. Viswanathan and G. Townsend, Inorg. Synth., 2006, 22, 101.

42 F. Hao, C. C. Stoumpos, P. Guo, N. Zhou, T. J. Marks, R. P. H. Chang and M. G. Kanatzidis, J. Am. Chem. Soc., 2015, 137, 11445.

43 T. A. Berhe, W.-N. Su, C.-H. Chen, C.-J. Pan, J.-H. Cheng, H.-M. Chen, M.-C. Tsai, L.-Y. Chen, A. A. Dubale and B.-J. Hwang, Energy Environ. Sci., 2016, 9, 323.

44 N. K. Noel, S. D. Stranks, A. Abate, C. Wehrenfennig, S. Guarnera, A.-A. Haghighirad, A. Sadhanala, G. E. Eperon, S. K. Pathak, M. B. Johnston, A. Petrozza, L. M. Herz and H. J. Snaith, Energy Environ. Sci., 2014, 7, 3061.

45 Q. Bao, X. Liu, S. Braun and M. Fahlman, Adv. Energy Mater., 2014, 4, 1301272.

46 Q. D. Yang, T.-W. Ng, M.-F. Lo, F. Y. Wang, N. B. Wong and C.-S. Lee, J. Phys. Chem. C, 2012, 116, 10982.

47 P. Peumans, A. Yakimov and S. R. Forrest, J. Appl. Phys., 2003, 93, 3693.

48 Z. R. Hong, Z. H. Huang and X. T. Zeng, Thin Solid Films, 2007, 515, 3019.

49 A. Guerrero, P. P. Boix, L. F. Marchesi, T. Ripolles-Sanchis, E. C. Pereira and G. Garcia-Belmonte, Sol. Energy Mater. Sol. Cells, 2012, 100, 185.

50 T. S. Glen, N. W. Scarratt, H. Yi, A. Iraqi, T. Wang, J. Kingsley, A. R. Buckley, D. G. Lidzey and A. M. Donald, Sol. Energy Mater. Sol. Cells, 2015, 140, 25.

51 C. M. Proctor, J. A. Love and T. Q. Nguyen, Adv. Mater., 2014, 26, 5957.

52 B. Lee, C. C. Stoumpos, N. Zhou, F. Hao, C. Malliakas, C. Yeh, T. J. Marks, M. G. Kanatzidis and R. P. H. Chang, J. Am. Chem. Soc., 2014, 136, 15379.

53 B. Saparov, J.-P. Sun, W. Meng, Z. Xiao, H.-S. Duan, O. Gunawan, D. Shin, I. G. Hill, Y. Yan and D. B. Mitzi, Chem. Mater., 2016, 28, 2315.

54 H. Yoshida, Anal. Bioanal. Chem., 2014, 406, 2231.

55 T. S. Glen, N. W. Scarratt, H. Yi, A. Iraqi, T. Wang, J. Kingsley, A. R. Buckley, D. G. Lidzey and A. M. Donald, J. Polym. Sci., Part B: Polym. Phys., 2016, 54, 216.

56 M.-C. Jung, S. R. Raga, L. K. Ono and Y. Qi, Sci. Rep., 2015, 5, 9863.

57 W. Nie, H. Tsai, R. Asadpour, A. J. Neukirch, G. Gupta, J. J. Crochet, M. Chhowalla, S. Tretiak, M. A. Alam and H. Wang, Science, 2015, 347, 522.

58 M. P. de Jong, L. J. van Ijzendoorn and M. J. A. de Voigt, Appl. Phys. Lett., 2000, 77, 2255.

59 S. Aharon, S. Gamliel, B. El Cohen and L. Etgar, Phys. Chem. Chem. Phys., 2014, 16, 10512. 